\title{
No evidence to allow quantification of optimum orthodontic force
}

\author{
What is the optimal force required for orthodontic tooth movement?
}

\section{Ren Y, Maltha JC, Kuijpers-Jagtman AM. Optimum force magnitude for orthodontic tooth movement: a systematic literature review. Angle Orthod 2003; 73:86-92}

Data sources Sources were MEDLINE (1966-December 2001) and hand searches of the main orthodontic and dental journals, along with bibliographies of selected articles.

Study selection Studies were excluded if there was no quantification of magnitude of force, rate or amount of movement, no control group, a split mouth design, fewer than five experimental sites, or if there was an observation period of less than 1 week. Neither were studies included that used functional or extra-oral appliances, or where medication and surgical or physical interventions other than orthodontic were used. Human and animal studies were included.

Data extraction and synthesis $A$ range of data relating to the forces applied to the tooth and their measurement were extracted from the studies identified. A large variation in data from current literature made it impossible to perform a meta-analysis.

Results Seventeen studies in animals and 12 in people were included. In eight human studies for canine retraction, initial forces of 800 $1500 \mathrm{cN}$ were used. Three studies of premolar tipping used forces from 50 to $200 \mathrm{cN}$, and two studies reported molar tipping forces of $100-500 \mathrm{cN}$.

Conclusions No evidence about the optimal force level in orthodontics could be extracted from literature. Well-controlled clinical studies and more standardised animal experiments in the orthodontic field would provide more insight into the relation between the force applied and the rate of tooth movement.

\section{Commentary}

The application of a force to a tooth results in tooth movement. This can occur pathologically, for example due to faulty restorative work or patient habit, but is usually thought of in the context of therapeutically applied orthodontic force. The fact that it occurs is beyond dispute and, indeed, thousands of orthodontists worldwide owe their existence to this fact. What is often debated is the concept of an "optimum" force. Ask any undergraduate about to sit their final examinations about orthodontic force and they will tell you that a force of $25 \mathrm{~g}$ is required to produce the optimum force to tip a tooth. This is achieved by activating the spring on a removable appliance two thirds of the way up the cusp of the tooth to be moved. Classical teaching, as proposed by Schwarz, ${ }^{1}$ defined optimal force as, "the force leading to a change in tissue pressure that approximated the capillary vessels blood pressure thus preventing their occlusion in the compressed periodontal ligament." According to Schwarz, lower forces would cause no reaction in the periodontal ligament leading to reduced or no movement. High forces would lead to areas of tissue necrosis preventing bone resorption and consequent tooth movement. The search for the optimal force is based on the hypothesis that a force of a certain magnitude and of certain characteristics (ie, continuous or intermittent) will produce the maximum tooth movement with minimum patient discomfort or damage.

The aim of this paper was to review the literature on orthodontic tooth movement in order to obtain a consensus on the optimum force. After applying the criteria listed in the study selection listed above, only 17 out of 161 animal studies and 12 out of 305 human studies could be included. Large variations in the studies made it impossible to perform the review as a meta-analysis. The authors discussed four main problems when comparing papers:

1. The difficulty in calculating the distribution of stresses and strains within the periodontal ligament.

2. Failure to control the precise nature of the tooth movement (ie, tipping versus bodily movement).

3. The concept that tooth movement can be divided into phases as described by Burstone. ${ }^{2}$ Some studies were only carried out for a short time. It could be that at the cellular level, therefore, inadequate time was allowed for movement to occur.

4. Large amounts of interindividual and intra-individual variations were noted.

There is therefore no agreement in the literature on what constitutes an optimum force level and, with respect to the fourth point above, it could be that the whole hypothesis, as first described by Schwarz, is flawed.

\section{Practice points}

There is currently no evidence available to quantify the magnitude of an optimum orthodontic force.

- The relationship between force and tooth movement has not been fully elucidated.

- The large individual variations seen in practice means that the magnitude of force required to move teeth can be variable.

\section{Nigel A Fox \\ Orthodontic Department, James Cook University Teaching \\ Hospital, Middlesbrough, Teeside, UK}

1. Schwarz AM. Tissue changes incident to orthodontic tooth movement. Int J Orthod. 1932; 18:331-352.

2. Burstone CJ. The biophysics of bone remodelling during orthodontics - optimal force considerations. In The Biology of Tooth Movement Edited by Norton LA, Burstone CJ. Boca Raton: CRC Press; 1989; 321-334.

Evidence-Based Dentistry (2003) 4, 80.

doi:10.1038/sj.ebd.6400222 